• 研究报告・

\title{
地锦苗在石灰岩土壤和红壤生境中 的繁殖成功的比较
}

\author{
黄至欢 ${ }^{1}$ 陆奇丰 ${ }^{1}$ 陈颖卓 $2^{*}$ \\ 1 (广西喀斯特植物保育与恢复生态学重点实验室, 广西壮族自治区中国科学院广西植物研究所, 广西桂林 541006) \\ 2 (湖南第一师范学院城南书院, 长沙 410205)
}

\begin{abstract}
摘要: 喀斯特地区石灰岩土壤和红壤间断分布的特点是影响该地区植物群落内物种组成的最重要环境因素之一。 为了研究不同生境中植物的繁殖对策及对生境的适应方式, 本文以生长在石灰岩土壤和红壤两种生境中的地锦苗 (Corydalis sheareri)为研究对象, 调查了两种生境中土壤的理化性质, 并开展了开花物候、花部特征、传粉者种类 和访问频率及繁殖成功的比较研究。结果显示, 石灰岩土壤中有机质、全氮、全钻的含量以及 $\mathrm{pH}$ 值均比红壤高, 而 土壤含水量则比红壤低。地锦苗的单花期在两种土壤生境中没有显著差异, 但石灰岩土壤中的地锦苗植株明显比 生长于红壤上的植株矮, 始花期比红壤中的推迟1周左右, 盛花期持续时间短, 并且提前进入末花期。花序大小、 花长、开口大小、距长及柱头直径在两种土壤生境中都没有显著差异。地锦苗的繁育系统为专性异交, 繁殖成功 依赖传粉者。访花者主要是以花蜜为报酬的黑颚条蜂(Anthophora melanognatha), 并且在两种生境中的访花频率没 有显著差异。两种生境中地锦苗的结䊏率没有差异, 但生长在石灰岩土壤上的地锦苗坐果率明显低于红壤上的。 我们的结果表明, 结籽率没有差异可能是由于传粉者的丰度不受生境影响, 导致两种生境中的地锦苗具有相同水 平的传粉服务; 而土壤性质的差异可能是导致两种生境中地锦苗花期物候和坐果率差异的重要原因。
\end{abstract}

关键词：地锦苗; 土壤类型; 花期物候; 繁育系统; 结䊏率; 坐果率; 繁殖成功

\section{Comparative study on reproductive success of Corydalis sheareri (Papav- eraceae) between alkaline limestone soil and red soil habitats in a karst area}

\author{
Zhihuan Huang ${ }^{1}$, Qifeng $\mathrm{Lu}^{1}$, Yingzhuo Chen ${ }^{2 *}$ \\ 1 Guangxi Key Laboratory of Plant Conservation and Restoration Ecology in Karst Terrain, Guangxi Institute of Botany, \\ Guangxi Zhuang Autonomous Region and Chinese Academy of Sciences, Guilin, Guangxi 541006 \\ 2 Chengnan Academy, Hunan First Normal University, Changsha 410205
}

\begin{abstract}
The discontinuous distribution between alkaline limestone soil with high calcium content and acidic red soil with low calcium content is characteristic of karst areas, and strongly affects plant species composition in natural communities. Whether the soil types affect plant reproductive success, however, remains unknown. Two populations of Corydalis sheareri, a species commonly occurring in alkaline limestone soil and acidic red soil, were investigated. The soil properties of the two habitats were examined, and flowering phenology, floral traits, floral visitor types and their behavior, breeding system and reproductive success were compared. Organic matter, total nitrogen, total calcium, and $\mathrm{pH}$ value were higher in the limestone soil, while soil moisture content was lower than that found in the red soil. Floral longevity was not significantly different between plants from the two habitats. However, plants found in the limestone soil were shorter in height and bloomed one week later with a shorter flowering period (4 weeks) than those found in the red soil (6 weeks). Floral traits (inflorescence size, flower length, opening size, spur length and stigma diameter)
\end{abstract}

收稿日期: 2017-06-02; 接受日期: 2017-09-04

基金项目：科技基础资源调查专项资助“中国西南地区极小种群野生植物调查与种质保存”(2017FY100100)、广西植物研究所基本业务费(GZY16003)、

广西喀斯特植物保育与恢复生态学重点实验室开放基金(1526222)

* 通讯作者 Author for correspondence. E-mail: yingzhuochen2011@163.com 
were not significantly different between the two habitats. Corydalis sheareri was obligately xenogamous, with sexual reproduction dependent on insect pollinators. The major floral visitor was Anthophora melanognatha, a long-tongued nectar collecting bee. Bee visit frequency and seed set per flower were not significantly different between the two habitats, but fruit set per plant in the limestone soil was significantly lower. Our results indicated that, no difference in the seed set could be explained by similar pollinator abundance, while the differences of flowering phenology and fruit set could be caused by differences in the soil properties between the two habitats.

Key words: Corydalis sheareri; soil property; flowering phenology; breeding system; fruit set; seed set; repr. oductive success

喀斯特地区因其特有的地理环境(石灰岩溶蚀 地貌)而具有较高的物种多样性(Yong et al, 2004)。 许多苔藓(Mohamed et al, 2005)和维管植物(Kiew, 1991, 2001; MacKinnon et al, 1996; IUCN, 1993)仅 分布于东南亚的石灰岩地区。例如, 马来西亚半岛 的喀斯特地区拥有 1,216 种被子植物(占马来西亚植 物总数的 $14 \%$ ), 其中 $21 \%$ 是马来半岛的特有物种, 11\%则仅限于喀斯特地区(Chin, 1977)。复杂的地形 (峭壁的裂隙和大量的洞穴)以及多变的环境使得喀 斯特地貌上能够提供大量的生态位来容纳较高的 物种多样性(Clements et al, 2006)。例如, 土层较厚 的天坑、沟渠和平缓山坡上可以维持如龙脑香科等 大树的生长, 而土层较薄的山丘顶部通常被草本植 物如苦苣苔科、凤仙花科和秋海棠属(Begonia) 等和 苔藓植物所占据(Kiew, 2001)。高比例的特有植物可 能是由于构造运动(地壳的隆升和海平面的升降 等)、地质作用(例如地下水对石灰岩长期蚀溶造成 的地表隆起和凹陷)、微生境隔离的程度(大量分散 的、土层厚度不同的岩缝和洞穴)以及遗传漂变等因 素产生(Yong et al, 2004; Clements et al, 2006; Shui et al, 2015)。

石灰岩土壤和红壤间断分布是喀斯特地区生 境的主要特征之一(曹建华等, 2003), 这一特征深刻 地影响了该地区的植物物种组成。石灰岩土层通常 较薄且具有较高浓度的 $\mathrm{Ca}^{2+}$ 、偏碱性以及较低的土 壤储水能力(曹建华等, 2003; 周卫和汪洪, 2007; Nie et al, 2011), 而红壤则具有较厚的土层且富含铁 铝氧化物、偏酸性以及较好的储水性(刘祖香等, 2013)。根据植物对石灰岩土壤的耐受程度可将喀斯 特地区的植物大致划分为钙生性植物和非钙生性 植物(屠玉麟, 1995; 周运超, 1997), 但这种简单的 分类方式很难准确地界定喀斯特地区复杂的生境,
也无法反映出植物对岩溶环境的适应策略。非专性 钙生植物为研究植物如何适应岩溶环境提供了独 特的材料, 并有利于通过对比研究检验植物的哪些 特征有利于植物适应岩溶环境, 从而有利于深入理 解喀斯特地区物种多样性的形成机制。

紫堇属(Corydalis)是罂粟科中最大的属, 约 428 种, 除北极地区外, 广布于北温带, 南至北非一印度 沙漠区的边缘, 个别种分布到东非的草原(吴征镒 等, 1996; Zhang et al, 2008)。我国的紫堇属植物有 298 种, 占全世界紫堇属物种数的 $69.6 \%$, 其中, 西 南部地区集中分布了大量该属特有种(吴征镒等, 1996; Zhang et al, 2008)。紫堇属植物的形态特征和 繁育系统在种间甚至种内都表现出较高的多样性, 因此吸引了较多繁殖生态学方面的研究(Higashi et al, 1988; 黎维平和刘胜祥, 1997; Zhang et al, 2009; 夏青等, 2012)。以往的研究发现, 一些紫堇属植物 对不同生境具有很强的适应性，它们既能够在酸性 红壤(基岩为砂页岩)上生长, 也能够在岩溶地区的 石灰岩钙质土壤上生长(钟济新, 1982)。针对同一物 种开展在两种土壤上的繁殖对策的对比研究, 可为 理解喀斯特地区植物物种多样性的形成机制提供 有价值的参考。

本研究旨在通过调查同一地区石灰岩土壤和 酸性红壤两种环境条件下的地锦苗 (Corydalis sheareri)的繁殖策略, 探讨环境因素对植物生长发 育的影响。主要从以下 4 个方面分析不同生境对植 物适合度的影响: (1)不同生境条件下的花期是否存 在差异？(2)不同生境条件下的花部特征是否存在 差异? (3)不同生境条件下的传粉者种类和访问频 率是否有差异? (4)不同生境条件下的繁殖成功是 否有差异? 通过回答上述问题, 探讨土壤环境对地 锦苗的繁育方式的影响, 以期为深入研究喀斯特地 
区植物多样性的形成及适应机制奠定基础。

\section{1 材料和方法}

\section{1 实验材料}

地锦苗是中国南方常见的早春开花的多年生 草本植物, 常见于沟边、路边、石山上或洞穴附近, 花期2-5月。以往的调查研究发现, 地锦苗既能够在 酸性红壤上生长, 也能够在石灰岩钙质土壤上生长 (图1A，B)。地锦苗的花冠由4片花瓣组成, 上花瓣延 伸形成存放花蜜的距, 匙形的下花瓣为蜂类访花者 提供了着陆平台, 两片相连的提琴形的内花瓣将花 药和柱头紧紧围住。当蜂类传粉者向下挤压内花瓣 吸取花蜜时, 花药和柱头暴露出来并接触传粉者。 此外, 和其他紫堇属植物一样(Maloof, 2000; Stolle, 2004; 王慧和李肖夏, 2017), 地锦苗的花具有花粉 次级呈现(secondary pollen presentation)的特点, 即 花粉从花药中散出后落置到柱头等非花药的花部 结构上，再由此结构呈现给传粉者。

实验地点分别选在位于广西桂林市雁山区的 桂林植物园 $\left(25^{\circ} 04^{\prime} 26^{\prime \prime} \mathrm{N}, 110^{\circ} 17^{\prime} 46^{\prime \prime} \mathrm{E}\right.$, 海拔 $\left.165 \mathrm{~m}\right)$ 和雁山园 $\left(25^{\circ} 04^{\prime} 40^{\prime \prime} \mathrm{N}, 110^{\circ} 18^{\prime} 23^{\prime \prime} \mathrm{E}\right.$, 海拔156 m), 其中桂林植物园的土壤类型为偏酸性红壤(为第四 纪红土发育而来的酸性土), 而雁山园的土壤类型 为石灰岩钙质土壤(母岩由碳酸盐岩类发育的一类 土壤)。我们在桂林植物园选取生于路边、湖边和围

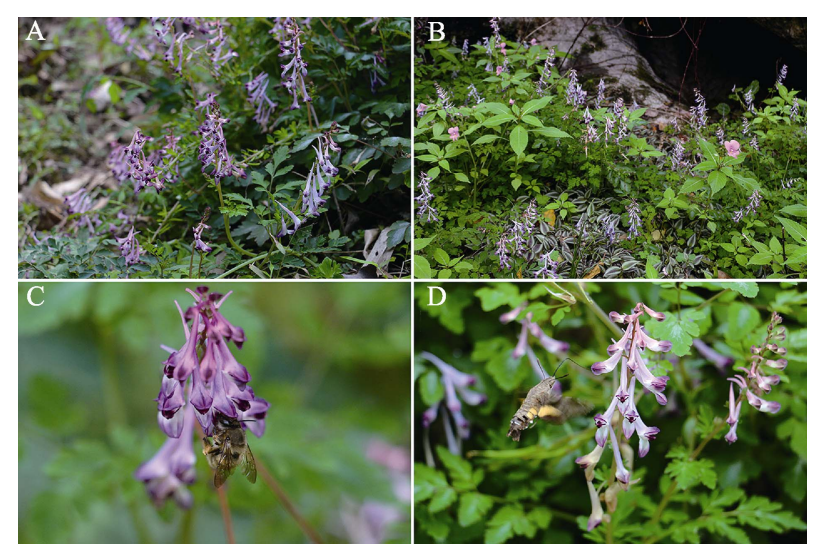

图 1 (A)在酸性红壤中生长的地锦苗; (B)在长有苔蘚的洞 穴外的碱性石灰岩土壤上生长的地锦苗; (C)黑颚条蜂访问 地锦苗; (D)长喙天蛾访问地锦苗。

Fig. 1 (A) Plants of Corydalis sheareri inhabited in the red soil (acidic); (B) Plants growing near the moist limestone caves with moss (calcareous); (C) A bee (Anthophora melanognatha) collecting nectar from C. sheareri; (D) A hawkmoth (Macroglossum corythus) sucking nectar from C. sheareri.
墙边的居群，在雁山园则选取生于石山、岩缝和洞 穴旁的居群作为研究对象。实验于2017年的2-5月 进行。实验材料均为野生自然种群, 常见的伴生开 花植物有大旗瓣风仙花(Impatiens macrovexilla)、 酢酱草(Oxalis corniculata)、韩信草(Scutellaria indica)等。

\section{2 土壤理化性质测定}

在桂林植物园和雁山园各选择 3 个地锦苗分布 地点, 每个地点随机取土壤样品 1 份, 用来测定土 壤养分等特性。称量土壤鲜重及土壤在 $105^{\circ} \mathrm{C}$ 条件 下烘干后的干重, 并计算土壤含水量。土壤含水量 $=($ 土壤鲜重 - 土壤干重 $) /$ 土壤鲜重。土壤有机质用 灰分法测定(黄宝强和安德军, 2013)。测定土壤中N、 $\mathrm{K} 、 \mathrm{P} 、 \mathrm{Ca}$ 的含量及 $\mathrm{pH}$ 值, 方法如下：N用凯氏定氮 法; K用火焰光度法; P用钼锑钕比色法; Ca用EDTA 容量法; pH用电位法(刘合霞等, 2017)。

\section{3 不同生境中的花期物候调查}

为了评价不同生境条件下的花期物候, 对地锦 苗的两种生境类型分别随机选取 1 个 $3 \mathrm{~m} \times 3 \mathrm{~m}$ 的样 方，于2017年2月 8 日至 4 月 26 日，每周调查开花植 株数。观察和统计的开花参数主要有始花期、盛花 期、末花期及各阶段持续时间，统计以花序为基本 单位。种群内总花序的 $10 \%$ 开花视为始花期, 达到总 花序的 $50 \%$ 及以上视为种群盛花期，总花序 $90 \%$ 的 花枯萎视为末花期, 所有花序都枯萎视为终花期。

为了确定不同生境中地锦苗的单花期, 从两种 生境中各 30 个花序的中部随机标记1朵花苞, 于 3 月 18-24日连续观察一周, 记录每朵花的开放状态。

\section{4 不同生境中花部特征比较}

为了确定不同生境中的开花特性，从两种生境 中分别随机选取 20 个未开放的花序, 于2017年3月 19-26日连续1周记录植株高度、花序大小和开花 顺序。

为了确定不同生境中植物的花部特征是否存 在差异, 分别从每个生境类型中各选取20朵中部位 置开放的花，用游标卡尺(精确度 $0.01 \mathrm{~mm})$ 测量花 冠长度(上花瓣顶端到距末端的距离)、开口大小 $($ 上 下花瓣顶端之间的距离)、距长度(花梗与花瓣连接 处到距末端的距离)和柱头直径(柱头的最大长度)。

\section{5 不同生境中的访花者及访问频率}

为了确定不同生境中地锦苗的传粉系统是否 有差异，于2017年3-4月，对每种生境类型各选取3 
个居群，每个居群选取10-15个花序作为观察对象。 以30 min为 1 个观察时间段, 于9:00-17:00期间, 详 细记录访花者的种类、访花次数、访花行为(获取的 报酬类型)等, 用于计算每种传粉者的访花频率。每 个居群累计观察不少于 10 个时间段。

每个传粉者类型捕捉2-3只, 保存在 $2 \mathrm{~mL}$ EP管 中, 硅胶干燥保存, 带回实验室鉴定物种。

\section{6 授粉实验}

为了确定地锦苗的繁育系统, 于 2017 年 4 月初 在桂林植物园进行了套袋和人工授粉处理。由于紫 堇属植物具有次级花粉呈现现象, 且花药和柱头距 离较近, 经常导致花药在花开放之前就已经落置在 柱头上, 因此在不损伤花的前提下的人工异交较为 困难(Maloof, 2000)。鉴于此, 我们的处理中不包括 异交授粉。随机选择20株植株, 每株上选择3个发育 状态相似的花序, 每个花序上又分别选取中部位置 的5朵未开放的花, 套袋以隔绝传粉者, 植株上其 余花朵不作任何处理。花朵开放后, 对每株植物的3 个花序分别进行以下处理: (1)套袋, 不作授粉处理, 用以检验是否存在自动自交; (2)人工辅助自交并套 袋, 用以检验是否自交亲和; (3)自然授粉, 作为对 照处理。每种处理用一种颜色的细线系在花梗上作 为标记, 约 20 天后, 统计每个处理的花序上的结果 数, 并从每个果序上随机选择 $1-2$ 个䕛果, 统计种 子数和败育胚珠数。

\section{7 不同生境中的结实率比较}

为了确定不同土壤环境是否会影响植物的繁 殖成功, 于2017年4月初分别在桂林植物园和雁山 园对地锦苗的两种生境类型各随机选择20个自然 授粉的植株, 用标牌各随机标记一个花序, 约 20 天 后, 统计果序上的坐果数, 并从每个果序上随机选 择 $1-2$ 个蒴果, 统计种子数和败育胚珠数。

\section{8 数据分析}

利用 R软件 ver. 3.1.0 (http://cran.r-project.org/) 的广义线性模型(GLM), 选择高斯分布与恒等连接 函数(Gaussian Distribution and Identity Link Function), 对生长在两种生境中的地锦苗的单花期、花 部特征、访花频率及繁殖成功进行差异分析。统计 数据采用平均值土标准误表示。

\section{2 结果}

\section{1 两种生境的土壤理化特性}

适合地锦苗生长的石灰岩土壤和红壤在土壤 理化性质上存在明显的差异。其中, 石灰岩土壤中 的有机质含量、全氮、全钻含量以及 $\mathrm{pH}$ 值均显著高 于红壤, 但土壤含水量明显低于红壤(表1)。

\section{2 不同生境对花期物候的影响}

石灰岩土壤上的地锦苗的始花期比红壤上的 推迟 1 周左右, 盛花期持续时间为 4 周, 比红壤上的 盛花期(6周)短, 并且提前进入末花期, 终花期则没 有差异(图2)。在红壤和石灰岩土壤上的单花期分别 为 $4.4 \pm 0.1$ 天和 $4.1 \pm 0.1$ 天, 两种生境下的单花期没 有显著差异 $(n=30, t=1.25, P=0.22)$ 。

\section{3 不同生境对开花特性的影响}

由表2可见, 相比于生长在红壤上的地锦苗, 生 长在石灰岩土壤上的植株高度明显更矮 $(n=20, t=$ $-2.08, P=0.04)$ 。总状花序的地锦苗的开花顺序为 自下往上依次开花。石灰岩土壤上的地锦苗单花序 花数 $(19.7 \pm 0.7)$ 与红壤上的 $(20.6 \pm 0.7)$ 没有显著差 异 $(n=20, t=-0.95, P=0.34)$ 。两种生境中的地锦苗 在花长、开口大小、距长和柱头直径上也没有明显 差异(表2)。

\section{4 不同生境中的传粉者及访问频率}

在开花期内连续观察发现, 地锦苗的访花昆虫

表 1 地锦苗两种生境中土壤特性的比较

Table 1 Comparisons of soil properties (mean \pm SE) between the limestone and red soil from the two habitats of Corydalis sheareri

\begin{tabular}{llllll}
\hline 土壤特性 Soil property & 样本量 Sample size & 石灰岩土壤 Limestone soil & 红壤 Red soil & $T$ & $P$ \\
\hline 含水量 Moisture (\%) & 3 & $21.73 \pm 0.98$ & $25.15 \pm 0.62$ & 2.95 & 0.04 \\
有机质 Organic matter (g/kg) & 3 & $61.10 \pm 1.36$ & $28.37 \pm 1.44$ & -18.36 & $<0.001$ \\
全氮 Total nitrogen (mg/kg) & 3 & $214.36 \pm 7.31$ & $87.46 \pm 5.89$ & -13.53 & $<0.001$ \\
全磷 Total phosphorus (mg/kg) & 3 & $11.09 \pm 0.81$ & $13.32 \pm 1.14$ & 1.52 & 0.20 \\
全钾 Total potassium (mg/kg) & 3 & $121.35 \pm 7.17$ & $110.06 \pm 6.45$ & -1.17 & 0.31 \\
全钻 Total calcium (g/kg) & 3 & $28.62 \pm 3.84$ & $3.18 \pm 1.50$ & -6.54 & 0.003 \\
$\mathrm{pH}$ & 3 & $7.75 \pm 0.12$ & $6.29 \pm 0.08$ & -10.27 & $<0.001$ \\
\hline
\end{tabular}




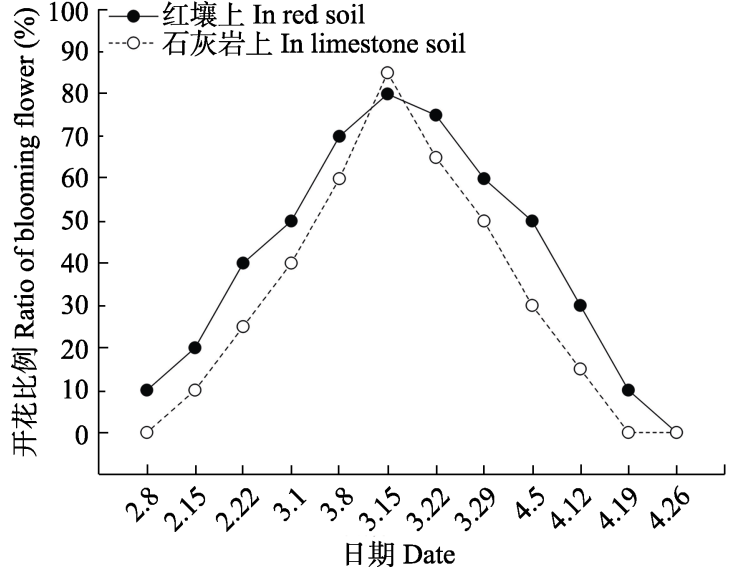

图 2 两种生境中地锦苗的花期物候

Fig. 2 Flowering phenology of Corydalis sheareri in the two habitats with different soil types

较为丰富, 两种生境中的主要传粉者都是黑颚条蜂 (Anthophora melanognatha), 它主要以花蜜为报酬, 访花时大多降落在下花瓣上, 通过挤压内花瓣后将 口器伸入距内吸取花蜜(图1C), 偶尔也会从上花瓣 末端咬开距来盗取花蜜。在正常访花过程中, 黑颚 条蜂的头部会紧压住雄荵, 在带走大量花粉的同时 给柱头带来部分异花花粉。黑颚条蜂的访问较为频
繁, 白天大部分时间均能观察到访花, 高峰期主要 集中在10:00-15:00, 受温度和光照的影响较为明 显。黑颚条蜂在两种生境条件下的访问频率没有显 著差异 $(t=1.44, P=0.15$, 表3, 图3)。我们只在红壤 生境中观察到中华蜜蜂(Apis cerana)访问地锦苗, 并且访问频率远低于同一观察样方内黑颚条蜂的 访问频率 $(P<0.001$, 图 3)。天蛾 (Macroglossum stellatarum)通常被认为是盗蜜者, 但我们的观察发 现，天蛾在吸取花蜜时，其喙上也携带有少量花粉 (图1D), 因此推测天蛾也具有一定的传粉作用。在 不同生境下, 天蛾的访问频率没有显著差异 $(t=$ $1.25, P=0.21$, 图3)。

\section{5 授粉实验}

实验结果显示，地锦苗的套袋和人工自花授粉 处理的花序均没有坐果(均为 $n=20)$, 表明其为自 交不亲和, 生殖成功完全依赖传粉者异花授粉。

\section{6 不同生境对繁殖成功的影响}

实验结果表明，地锦苗在酸性红壤上自然授粉 的坐果率为 $54.7 \% \pm 4.5 \%(n=20)$, 结籽率为 $70.6 \%$ $\pm 2.3 \%(n=30)$ 。相比之下，石灰岩土壤上生长的地 锦苗的坐果率 $(41.4 \% \pm 4.1 \%, n=20)$ 明显更低 $(t=$

表 2 两种生境中地锦苗的花部特征比较

Table 2 Comparisons of floral traits of Corydalis sheareri from the two habitats with different soil types

\begin{tabular}{llllll}
\hline 花部特征 Floral characters & 样本量 Sample size & 红壤 Red soil & 石灰岩土壤 Limestone soil & $T$ & $P$ \\
\hline 植株高度 Plant height (cm) & 20 & $34.8 \pm 1.8$ & $30.2 \pm 1.3$ & -2.08 & 0.04 \\
花数/花序 Flower number/inflorescence & 20 & $20.6 \pm 0.7$ & $19.7 \pm 0.7$ & -0.95 & 0.34 \\
花长 Floral length (mm) & 20 & $27.96 \pm 0.24$ & $27.39 \pm 0.22$ & -1.76 & 0.09 \\
开口大小 Opening size (mm) & 20 & $13.02 \pm 0.27$ & $12.45 \pm 0.23$ & -1.62 & 0.11 \\
距长 Spur length (mm) & 20 & $17.45 \pm 0.15$ & $17.12 \pm 0.17$ & -0.98 & 0.34 \\
柱头直径 Stigma diameter (mm) & 20 & $1.54 \pm 0.01$ & $1.49 \pm 0.02$ & -1.86 & 0.07 \\
\hline
\end{tabular}

表 3 两种生境中地锦苗的访花者类型和访问频率比较。统计使用 GLM 分析, 同一列不同字母代表同种访花者在不同居群 间差异显著 $(P<\mathbf{0 . 0 5})$ 。

Table 3 Floral visitor types and their visitation frequency to Corydalis sheareri in the two habitats. The different letters for the same column indicate visit frequency was significantly different between plots for the same floral visitor at the level of $P<0.05$ by GLM (Gaussian distribution and identity link function).

\begin{tabular}{|c|c|c|c|c|c|}
\hline \multirow{2}{*}{$\begin{array}{l}\text { 土壤类型 } \\
\text { Soil type }\end{array}$} & \multirow{2}{*}{$\begin{array}{l}\text { 样地 } \\
\text { Plot }\end{array}$} & \multirow{2}{*}{$\begin{array}{l}\text { 观察时间段 } \\
\text { Observed censuses }(0.5 \text { h) }\end{array}$} & \multicolumn{3}{|c|}{ 访问次数/花数-小时 Visits/flowers.h } \\
\hline & & & 条蜂 Anthophora & 蜜蜂 Honeybee & 天蛾 Sphingid \\
\hline \multirow{3}{*}{$\begin{array}{l}\text { 红壤 } \\
\text { Red soil }\end{array}$} & 池塘边 Poolside & 14 & $0.19 \pm 0.02^{\mathrm{ab}}$ & $0^{\mathrm{b}}$ & $0.10 \pm 0.03^{\mathrm{ab}}$ \\
\hline & 路边 Roadside & 16 & $0.16 \pm 0.03^{\mathrm{ab}}$ & $0.07 \pm 0.02^{\mathrm{a}}$ & $0^{c}$ \\
\hline & 苗圃角落 Yard's corner & 16 & $0.24 \pm 0.03^{\mathrm{a}}$ & $0.11 \pm 0.02^{\mathrm{a}}$ & $0.16 \pm 0.04^{\mathrm{a}}$ \\
\hline \multirow{3}{*}{$\begin{array}{l}\text { 石灰岩土壤 } \\
\text { Limestone soil }\end{array}$} & 洞穴外 Near the cave & 12 & $0.20 \pm 0.04^{\mathrm{ab}}$ & $0^{\mathrm{b}}$ & $0.05 \pm 0.03^{\mathrm{b}}$ \\
\hline & 石缝处 Stony crevices & 16 & $0.11 \pm 0.03^{\mathrm{b}}$ & $0^{\mathrm{b}}$ & $0^{\mathrm{c}}$ \\
\hline & 石山小径 Stony pathlet & 16 & $0.17 \pm 0.04^{\mathrm{ab}}$ & $0^{\mathrm{b}}$ & $0.12 \pm 0.04^{\mathrm{ab}}$ \\
\hline
\end{tabular}


$\square$ 条蜂 Anthophora $\mathbb{\mathbb { N }}$ 天蛾 Sphingid $\square$ 蜜蜂 Honeybee

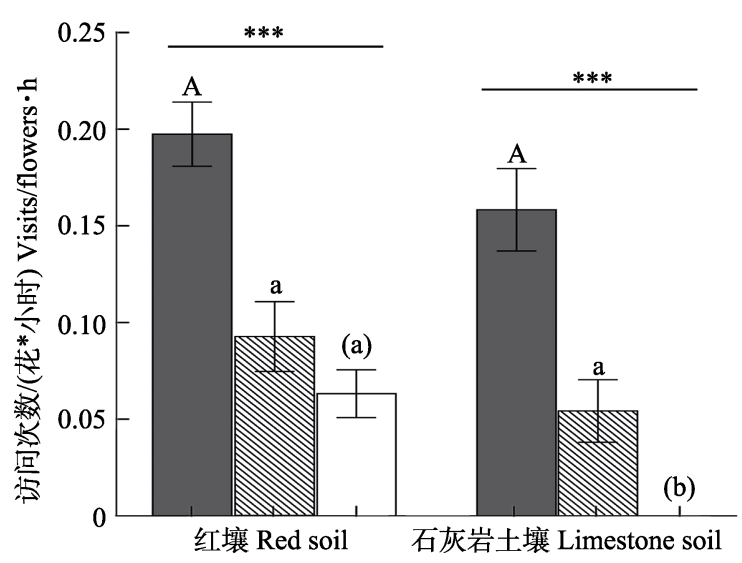

图 3 两种生境中地锦苗的 3 种访花者的访问频率比较。相 同的字母(分大小写)表示同种访花者在两种土壤生境中的 访问频率没有差异, 不同字母表示同种访花者在两种土壤 生境中的访问频率具有明显差异; *表示同种土壤生境中黑 颚条蜂与其他两种访问者的访问频率均具有显著差异。

Fig. 3 Comparisons of visit frequency among the three floral visitors in the two habitats. The same letter (case-sensitive) means no significant difference between the same floral visitor, while different letters mean significant difference of the same floral visitor between the two habitats; * mean visitation frequency of Anthophora melanognatha significantly higher than the other two floral visitors.

2.18, $P=0.04)$; 而石灰岩土壤上的地锦苗的结籽率 $(66.9 \% \pm 2.3 \%, n=30)$ 与红壤上的结籽率之间则没 有差异 $(t=1.16, P=0.25)$ 。

\section{3 讨论}

\section{1 不同生境对花期的影响}

植物的开花物候和单花期是植物生命活动对 时间变化的反应(Rathcke \& Lacey, 1985)。一般认为, 海拔、纬度和气温等环境因素的变化会导致植物花 期物候的改变(Olsson \& Ågren, 2002)。例如, 通过 同质园实验, Olsson和Ågren (2002)对多年生草本植 物千屈菜(Lythrum salicaria)不同纬度来源的居群物 候研究发现, 来源于高纬度地区的千屈菜开花早并 且花期短。植物的花期物候对土壤环境因素(诸如土 壤养分和水分等)的变化也会产生不同程度的反应, 杨期和等(2003)对不同生境中的薇甘菊(Mikania micrantha) 的开花结实观测表明, 土壤肥力较高, 薇甘菊的开花数量多, 花期相应较长; 而对青藏高 原东部高寒草甸封育和施肥生境中18种植物的物 候对比研究表明, 施肥对双子叶植物的物候没有显 著影响(巴雅尔塔等, 2010)。植物为了在水分胁迫环
境中生存通常会加速发育进程(Kazan \& Lyons, 2016)。McMaster和Wilhelm (2003)发现在较为严重 的水分胁迫条件下, 小麦的开花期和成熟期分别比 对照处理(充分灌溉)提前 13 天和 15 天, 最终导致整 个生育期缩短。对亲缘关系较远的3种一年生草本 植物的水分胁迫控制实验结果同样表明, 水分胁迫 导致花期有不同程度的提前, 并且花期持续时间缩 短(Aronson et al, 1992)。但是, 多年生植物在水分胁 迫环境下开花物候的观察结果往往与针对一年生 草本植物的研究结果相悖。戴永丽(2014)对不同生 境中小甘菊(Cancrinia discoidea)的物候观测发现, 在土壤含水量高的生境中, 小甘菊的花期比土壤含 水量低的生境中的花期提前5-6天。我们对两种生 境中多年生草本地锦苗的花期调查表明, 石灰岩钙 土上的地锦苗的始花期比酸性红壤上的推迟, 末花 期提前, 盛花期持续时间缩短(图2)。本研究选取的 地锦苗的两种生境间相距不足 $2 \mathrm{~km}$, 海拔和气温均 没有差异; 而土壤肥力和含水量具有明显差异, 导 致石灰岩生境中的地锦苗的营养生长阶段延长, 开 花期缩短, 最终导致整个生育期缩短。这说明石灰 岩土壤生境对地锦苗的开花物候具有明显的胁迫 作用。

\section{2 不同生境对花部特征的影响}

花部特征不仅决定有性繁殖(传粉与受精等)的 成功率(Waser \& Price, 1983)及后代遗传组成和适 合度(Conner et al, 1996; Thomson, 2006), 同时也决 定其对外界自然干扰(如降雨或干旱)抵抗能力的强 弱(Herrera, 2005; Fan et al, 2012), 因此, 许多植物 在不同生境中的花部特征常常存在显著差异。例如, 对两种生境中蝴蝶花(Iris japonica)的花部特征的比 较发现, 相比于林缘旷地生境, 竹林生境中的蝴蝶 花具有大而轻的花冠(王永健等, 2010)。对四川黄龙 沟两种生境中的少花鹤顶兰(Phaius delavayi)的花 部特征比较发现, 森林生境中的个体与钙化滩地中 的个体相比, 唇瓣长和宽没有差异, 但具有更长的 距(黄宝强和安德军, 2013)。对于以异交为主的植物 而言, 处于不同生境的种群还可能面临传粉者类群 和组成的差异, 尤其是异交物种扩散到一个新分布 区域的先锋种群必然经历着适应局部“更有效传粉 者”的强烈选择(Johnson \& Steiner, 1997; Fortuna et al, 2008), 因此, 处于不同生境的种群可能出现适 应局部传粉者的花部特征调整(Robertson \& Wyatt, 
1990; van Kleunen et al, 2007; Huang \& Shi, 2013)。 我们对两种生境下的地锦苗的花部特征测量结果 表明, 酸性红壤和石灰岩土壤上生长的地锦苗在花 序大小、花长、开口大小、距长和柱头直径上都没 有差异, 这可能与两种生境中的地锦苗受到相同水 平的传粉者选择压力有关。

\section{3 不同生境对传粉者行为的影响}

特化的花部特征使得仅有少数访花昆虫可以 为紫堇属植物提供传粉服务, 但大多数研究也发现 紫堇属植物的访花昆虫专一性并不强, 体型大小和 访花行为相似的熊蜂、蜜蜂和切叶蜂(Megachile)等 长吻类型的蜂类都可以成为其有效传粉者 (Ohara \& Higashi, 1994; Kudo et al, 2001; Wang et al, 2014; 王慧和李肖夏, 2017)。王慧(2014)对分布于华中地 区的地锦苗的野外访花者观察发现, 切叶蜂和熊蜂 (主要是Bombus richardsi) 是其主要传粉者; 我们对 分布于华南地区的地锦苗的访花者观察发现, 访花 者主要是以花蜜为报酬的黑颔条蜂(图1C), 进一步 证实了不同地域的传粉者可能具有多样性。在两种 生境中地锦苗的访花频率并没有明显差异(图3), 结 果说明其传粉者丰度可能不受生境的影响, 这也是 专性异交的地锦苗能够适应两种土壤生境的重要 原因。

\section{4 不同生境对繁殖成功的影响}

植物在新生境中扩散的必要条件是它们能够 在新生境中成功繁殖(Richardson et al, 2000)。在只 有少数个体被引入新生境且传粉受到限制的情况 下, 与自交亲和相结合的自动自交经常被认为是重 要的繁殖保障策略(Baker, 1955, 1967)。相比之下, 专性异交则对传粉者具有完全的依赖性, 并且其后 代具有更高的遗传多样性(Ruhsam et al, 2013)。对两 种土壤类型的多个居群的地锦苗的传粉观察结果 表明, 两种土壤上的传粉者种类相同, 并且访问频 率也没有差异。自然授粉条件下的两种生境类型的 结籽率没有差异, 说明在传粉者访花频率没有差异 的前提下, 地锦苗的雌性功能不受环境影响。而石 灰岩生境中的地锦苗的坐果率明显比酸性红壤上 的低, 植株也明显比红壤生境中的矮(表2), 我们推 测石灰岩土壤上的地锦苗的繁殖成功可能存在着 明显的资源限制。

致谢：感谢广西壮族自治区中国科学院广西植物研
究所的刘静女士对植物标本进行鉴定; 感谢两位匿 名审稿专家和责任编委提出的修改建议!

\section{参考文献:}

Aronson J, Kigel J, Shmida A, Klein J (1992) Adaptive phenology of desert and Mediterranean populations of annual plants grown with and without water stress. Oecologia, 89, 17-26.

Baker HG (1955) Self-compatibility and establishment after "long-distance" dispersal. Evolution, 9, 347-349.

Baker HG (1967) Support for Baker's law-as a rule. Evolution, 21, 853-856.

Bayaerta, Jia P, Yang X, Du GZ (2010) Response of dominating species flowering phenology to fertilization in Qinghai-Tibetan alpine meadow. Acta Prataculturae Sinica, 19, 233-239. (in Chinese with English abstract) [巴雅尔塔, 贾 鹏, 杨晓, 杜国祯 (2010) 青藏高原高寒草甸组分种花期 物候对施肥响应. 草业学报, 19, 233-239.]

Cao JH, Yuan DX, Pan GX (2003) Some soil features in karst ecosystem. Advance in Earth Sciences, 18, 37-44. (in Chinese with English abstract) [曹建华, 袁道先, 潘根兴 (2003) 岩溶生态系统中的土壤. 地球科学进展, 18, 37-44.]

Chin SC (1977) The limestone hill flora of Malaya: Part 1. Gardens’ Bulletin Singapore, 30, 165-220.

Clements R, Sodhi NS, Schilthuizen M, Ng PK (2006) Limestone karsts of Southeast Asia: imperiled arks of biodiversity. AIBS Bulletin, 56, 733-742.

Conner JK, Rush S, Jennetten P (1996) Measurements of natural selection on floral traits in wild radish (Raphanus raphanistrum). I. Selection through lifetime female fitness. Evolution, 50, 1127-1136.

Dai YL (2014) The influence of different habitat and planting density on growth development and ornamental value of Cancrinia discoidea (Ledeb.) Poljak. Master dissertation. Xinjiang Agricultural University, Urumchi. (in Chinese with English abstract) [戴永丽 (2014) 不同生境与栽培密度对 小甘菊生长发育与观赏价值的影响. 新疆农业大学硕士 学位论文, 乌鲁木齐.]

Fan XL, Barrett SCH, Lin H, Chen LL, Zhou X, Gao JY (2012) Rain pollination provides reproductive assurance in a deceptive orchid. Annals of Botany, 110, 953-958.

Fortuna MA, García C, Guimarães PR Jr, Bascompte J (2008) Spatial mating networks in insect-pollinated plants. Ecology Letters, 11, 490-498.

Herrera J (2005) Flower size variation in Rosmarinus officinalis: individuals, populations and habitats. Annals of Botany, 95, 431-437.

Higashi S, Ohara M, Arai H, Matsuo K (1988) Robber-like pollinators: overwintered queen bumblebees foraging on Corydalis ambigua. Ecological Entomology, 13, 411-418.

Huang BQ, An DJ (2013) Impacts of microenvironment on 
pollination success of an orchid species Phaius delavayi in Huanglong Valley, Sichuan. Bulletin of Botanical Research, 33, 80-85. (in Chinese with English abstract) [黄宝强, 安 德军 (2013) 两种生境对四川黄龙沟少花鹤顶兰有性繁 殖成功的影响. 植物研究, 33, 80-85.]

Huang SQ, Shi XQ (2013) Flower isolation in Pedicularis: how do congeners with shared pollinators minimize reproductive interference? New Phytologist, 199, 858-865.

IUCN (1993) Parks for Life: Report of the IVth World Congress on National Parks and Protected Areas. The World Conservation Union, Gland, Switzerland.

Johnson SD, Steiner KE (1997) Long-tongued fly pollination and evolution of floral spur length in the Disa draconis complex (Orchidaceae). Evolution, 51, 45-53.

Kazan K, Lyons R (2016) The link between flowering time and stress tolerance. Journal of Experimental Botany, 67, 47-60.

Kiew R (1991) The limestone flora. In: The State of Nature Conservation in Malaysia (ed. Kiew R), pp. 42-50. Malayan Nature Society, Kuala Lumpur.

Kiew R (2001) Towards a limestone flora of Sabah. Malayan Nature Journal, 55, 77-93.

Kudo G, Maeda T, Narita K (2001) Variation in floral sex allocation and reproductive success within inflorescences of Corydalis ambigua (Fumariaceae): pollination efficiency or resource limitation? Journal of Ecology, 89, 48-56.

Li WP, Liu SX (1997) Reproductive ecology of Corydalis sheareri var. bulbillifera (Papaveraceae). I. Pollination ecology. Journal of Central China Normal University (Natural Sciences Edition), 31, 87-91. (in Chinese with English abstract) [黎维平, 刘胜祥 (1997) 珠芽紫堇的生殖生态 学研究. I. 传粉生态学. 华中师范大学学报(自然科学版), 31, 87-91.]

Liu HX, Li B, Hu XH, Deng T, Huang SX (2017) A study on adaptation of 3 species of Gesneriaceae in limestone soil and red soil. Guihaia, doi:10.11931/guihaia.gxzw201612011. (in Chinese with English abstract) [刘合霞, 李博, 胡兴华, 邓 涛, 黄仕训 (2017) 三种苦苣苔对石灰土和红壤的适应性 分析. 广西植物, doi:10.11931/guihaia.gxzw201612011.]

Liu ZX, Chen XM, Jing Y, Huang QR, Li QX (2013) Hydraulic characteristics and its impact factors in typical red soil region. Bulletin of Soil and Water Conservation, 33, 21-25. (in Chinese with English abstract) [刘祖香, 陈效民, 靖彦, 黄欠如, 李秋霞 (2013) 典型旱地红壤水力学特性及其 影响因素研究. 水土保持通报, 33, 21-25.]

MacKinnon K, Hatta G, Halim H, Mangalik A (1996) The Ecology of Kalimantan. Periplus Editions, Singapore.

Maloof JE (2000) Reproductive biology of a North American subalpine plant: Corydalis caseana A. Gray ssp. brandegei (S. Watson) GB Ownbey. Plant Species Biology, 15, 281-288.

McMaster GS, Wilhelm WW (2003) Phenological responses of wheat and barley to water and temperature: improving simulation models. The Journal of Agricultural Science, 141,
141, 129-147.

Mohamed H, Yong KT, Damanhuri A, Latiff QA (2005) Moss diversity of Langkawi Islands, Peninsular Malaysia. Malayan Nature Journal, 57, 243-254.

Nie YP, Chen HS, Wang KL, Tan W, Deng PY, Yang J (2011) Seasonal water use patterns of woody species growing on the continuous dolostone outcrops and nearby thin soils in subtropical China. Plant and Soil, 34, 399-412.

Ohara M, Higashi S (1994) Effects of inflorescence size on visits from pollinators and seed set of Corydalis ambigua (Papaveraceae). Oecologia, 98, 25-30.

Olsson K, Ågren J (2002) Latitudinal population differentiation in phenology, life history and flower morphology in the perennial herb Lythrum salicaria. Journal of Evolutionary Biology, 15, 983-996.

Rathcke B, Lacey EP (1985) Phenological patterns of terrestrial plants. Annual Review of Ecology and Systematics, 16, 179-214.

Richardson DM, Pyšek P, Rejmanek M, Barbour MG, Panetta FD, West CJ (2000) Naturalization and invasion of alien plants: concept and definitions. Diversity and Distributions, 6, 93-107.

Robertson JL, Wyatt R (1990) Evidence for pollination ecotypes in the yellow fringed orchid, Platanthera ciliaris. Evolution, 44, 121-133.

Ruhsam M, Hollingsworth PM, Ennos RA (2013) Patterns of mating, generation of diversity, and fitness of offspring in a Geum hybrid swarm. Evolution, 67, 2728-2740.

Shui W, Chen YP, Wang YW, Su ZA, Zhang S (2015) Origination, study progress and prospect of karst Tiankeng research in China. Acta Geographica Sinica, 70, 431-446. [税 伟, 陈毅萍, 王雅文, 苏正安, 张素 (2015) 中国喀斯特 天坑研究: 起源、进展与展望. 地理学报, 70, 431-446.]

Stolle J (2004) Biological flora of Central Europe: Corydalis pumila (Host) Rchb. Flora, 199, 204-217.

Thomson JD (2006) Tactics for male reproductive success in plants: contrasting insights of sex allocation theory and pollen presentation theory. Integrative and Comparative Biology, 46, 390-397.

Tu YL (1995) An analysis of flora and ecological characteristics of karst scrubs in Guizhou Province. Journal of Guizhou Normal University (Natural Sciences), 13(3), 1-8. (in Chinese with English abstract) [屠玉麟 (1995) 贵州喀斯特灌 丛区系与生态特征分析. 贵州师范大学学报(自然科学 版), 13(3), 1-8.]

van Kleunen M, Manning JC, Pasqualetto V, Johnson SD (2007) Phylogenetically independent associations between autonomous self-fertilization and plant invasiveness. The American Naturalist, 171, 195-201.

Wang H (2014) Divergence in floral traits under the selection of pollinators in sympatric Corydalis species. $\mathrm{PhD}$ dissertation. Wuhan University, Wuhan. (in Chinese with English abstract) [王慧 (2014) 同域分布紫堇属植物传粉选择压 
力下的花部特征分化, 武汉大学博士学位论文, 武汉.]

Wang H, Li XX (2017) Differentiation in breeding system and pollination of three sympatric Corydalis species. Plant Science Journal, 35, 186-193. (in Chinese with English abstract) [王慧, 李肖夏 (2017) 同域分布的紫堇属三种植 物的繁育系统和传粉差异. 植物科学学报, 35, 186-193.]

Wang H, Tie S, Yu D, Guo YH, Yang CH (2014) Change of floral orientation affects pollinator behavior and pollination efficiency in a bee-pollinated plant, Corydalis sheareri. PLoS ONE, 9, e95381.

Wang YJ, Fang X, Zhang ZC (2010) Effect of different habitats on floral, fruit and seed traits of Iris japonica Thunb. Acta Ecologica Sinica, 30, 4628-4635. (in Chinese with English abstract) [王永健, 方兴, 钟章成 (2010) 不同生境对蝴蝶 花花部与果实特征的影响. 生态学报, 30, 4628-4635.]

Waser NM, Price MV (1983) Pollinator behaviour and natural selection for flower colour in Delphinium nelsonii. Nature, 302, 422-424.

Wu ZY, Zhuang X, Su ZY (1996) The systematic evolution of Corydalis in relation to florogenesis and floristic regionalization in the world. Acta Botanica Yunnanica, 18, 241-268. (in Chinese with English abstract) [吴征镒, 庄璇, 苏志云 (1996) 论紫堇属的系统演化与区系发生和区系分区的关 系. 云南植物研究, 18, 241-268.]

Xia Q, Zhou SB, Zhang D, Chao TC (2012) Floral syndrome and breeding system of Corydalis edulis. China Journal of Chinese Materia Medica, 37, 1191-1196. (in Chinese with English abstract) [夏青, 周守标, 张栋, 晁天彩 (2012) 紫 堇的花部综合特征与繁育系统的研究. 中国中药杂志, 37, 1191-1196.]

Yang QH, Feng HL, Ye WH, Cao HL, Deng X, Xu KY (2003) An investigation of the effects of environmental factors on the flowering and seed setting of Mikania micrantha H. B. K
(Compositae). Journal of Tropical and Subtropical Botany, 11, 123-126. (in Chinese with English abstract) [杨期和, 冯惠玲, 叶万辉, 曹洪麟, 邓雄, 许凯扬 (2003) 环境因 素对薇甘菊开花结实影响初探. 热带亚热带植物学报, 11, 123-126.]

Yong HS, Ng FSP, Lee EYE (2004) Sarawak bau limestone biodiversity. Sarawak Museum Journal, 80(6), 105-146.

Zhang ML, Su ZY, Lidén M, Grey-Wilson C (2008) Papaveraceae. In: Flora of China (eds Wu ZY, Raven PH, Hong DY), Volume 7, Menispermaceae through Capparaceae. Science Press, Beijing \& Missouri Botanical Garden Press, St. Louis.

Zhang YW, Yu Q, Zhao JM, Guo YH (2009) Differential effects of nectar robbing by the same bumble-bee species on three sympatric Corydalis species with varied mating systems. Annals of Botany, 104, 33-39.

Zhong JX (1982) Flora Atlas of Limestone Plants in Guangxi. Guangxi People's Publishing House, Nanning. (in Chinese) [钟济新 (1982) 广西石灰岩石山植物图谱. 广西人民出 版社, 南宁.]

Zhou W, Wang H (2007) The physiological and molecular mechanisms of calcium uptake, transport, and metabolism in plants. Chinese Bulletin of Botany, 24, 762-778. (in Chinese with English abstract) [周卫, 汪洪 (2007) 植物钙吸收、转 运及代谢的生理和分子机制. 植物学通报, 24, 762-778.]

Zhou YC (1997) A study on the part plants' main nutrient elements content of Guizhou Karst region. Journal of Guizhou Agricultural College, 16(1), 11-16. (in Chinese with English abstract) [周运超 (1997) 贵州喀斯特植被主要营养元素 含量分析. 贵州农学院学报, 16(1), 11-16.]

(责任编委：黄双全 责任编辑：时意专) 